\title{
Contextual recommender systems using a multidimensional approach
}

\author{
Mohammed Mahmudur Rahman \\ Lecturer, Dept. of Computer Science \& Engineering, International Islamic University, Chittagong, Bangladesh
}

Email address:

mmr@cse.iiuc.ac.bd

\section{To cite this article:}

Mohammed Mahmudur Rahman. Contextual Recommender Systems Using a Multidimensional Approach. International Journal of Intelligent Information Systems. Vol. 2, No. 4, 2013, pp. 55-63. doi: 10.11648/j.ijiis.20130204.11

\begin{abstract}
Recommender systems use the past experiences and preferences of the target users as a basis to provide personalized recommendations for them and as the same time, solve the information overloading problem. Context as the dynamic information describing the situation of items and users and affecting the user's decision process is essential to be used by recommender systems. Multidimensional approach to recommender systems that can provide recommendations based on additional contextual information besides the typical information on users and items used in most of the current recommender systems. This approach supports multiple dimensions, profiling information, and hierarchical aggregation of recommendation. The recommender system could simultaneously possess the advantages of content-based recommendation, knowledge-based recommendation, collaborative filtering recommendation and On-Line Analytical Processing (OLAP) in segmenting the information. Following the improvement of the recommendation structure, it doesn't have to limit its analysis on the user and product to compute for the recommendation result and it could also handle and determine more complex contextual information as recommendation computation foundation. It could develop better results if applied in different domains. This work extends the multidimensional recommendation model concept of Adomavicius and Tuzhilin (2001) and proposes a multidimensional recommendation environment to integrate the contextual information.
\end{abstract}

Keywords: Aggregation, Contextual, Multidimensional, Recommendation

\section{Introduction}

The recommender system is not only limited to E-commerce. It is also applicable for searching the most appropriate results in various search systems. Recommender system has already been developed for many years and there were many researches on the improvements of traditional recommendation methods. Majority of the researches focused on the improvement of the algorithm and combined applications of traditional recommender systems but they all have a similar drawback. Majority of the recommender systems use the gathered data under similar environment to provide recommendations. It was discovered, in an actual experience, that if only user's past behaviors were considered and the contextual information were ignored, it often caused suspicion in the recommendation results. In recent years, there were also numerous studies proving that, to improve a recommender system, it should all begin in data collection and profile establishment. In the inferring process, the effects of contextual information should also be considered and be used as the criterion of recommendation to provide appropriate recommendation results. The multidimensional recommendation model (MD recommendation model) proposed by Adomavicius and Tuzhilin (2001) as the foundation to establish a recommendation structure with multidimensional data collection and analysis ability and solve the movie recommendation problems with the use of hierarchy processing and aggregate calculating capabilities.

\section{Recommender Systems}

Traditionally, recommender systems deal with applications that have two types of entities, users and items. The recommender system would first acquire the ratings of users toward items they have already experienced to analyze their interests and preferences then provide recommendations from items of the same classification that haven't been rated by the users. In other words, traditional 
recommender system could be shown as the values of two-dimensional "Users $\times$ Items" matrix and it also computed the rating function of all the users toward the items $\mathrm{R}(\mathrm{u}, \mathrm{i})$. It can be shown as $R$ : Users $\times$ Items $\rightarrow$ Ratings.

According to Balabanovic and Shoham [1997], the approaches to recommender systems are usually classified as Content-based, Collaborative, and Hybrid.

All of the approaches focus on recommending items to users or users to items and do not take into consideration additional contextual information, such as time, place, the company of other people, and other factors affecting recommendation experiences. To address these issues, Adomavicius and Tuzhilin proposed a multidimensional approach to recommendations where the traditional two-dimensional user/item paradigm was extended to support additional dimensions capturing the context in which recommendations are made. This multidimensional approach is based on the multidimensional data model used for data warehousing and On-Line Analytical Processing applications in databases, on hierarchical aggregation capabilities, and on user, item and other profiles defined for each of these dimensions. Here also mention how the standard multidimensional OLAP model is adjusted when applied to recommender systems. Finally, to provide more extensive and flexible types of recommendations that can be requested by the user on demand, Adomavicius and Tuzhilin present a Recommendation Query Language (RQL) that allows users to express complex recommendations that can take into account multiple dimensions, aggregation hierarchies, and extensive profiling information.

\section{Multidimensional Recommendation Structure}

The proposed multidimensional recommendation structure of this study is shown in figure 1 . The multidimensional recommendation is used as a foundation to establish a complete multidimensional recommendation model. The components and process of the structure will be elaborately discussed below.

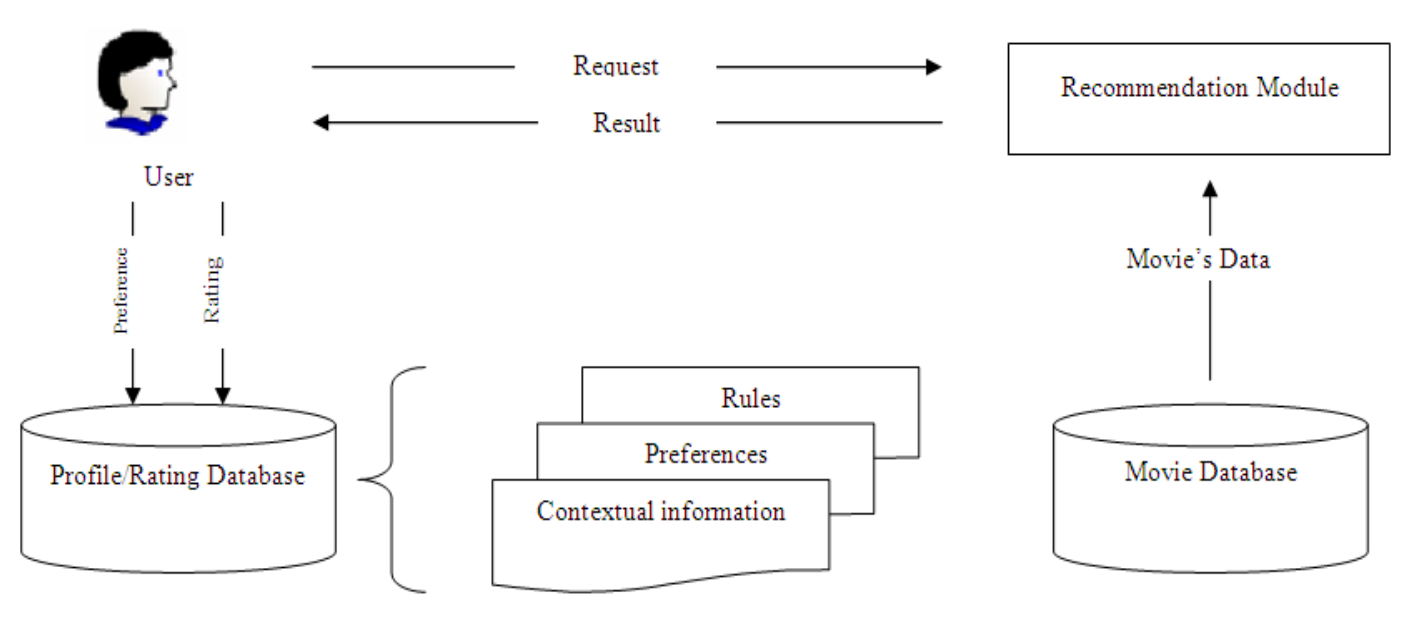

Figure1. Multidimensional Recommendation Structure

\subsection{Recommendation Module}

Since the ratings of users toward an item might belong to different hierarchies, the recommender system uses the multidimensional recommendation foundation to forecast the unknown rating. Here uses the concept of content-based recommendation to establish the dimension profile. Each dimension profile includes the attribute properties and its weighted value. Higher weighted value indicates the more important the attribute is. In the first stage, the recommendation module compares the user profile (preferences and rules) and the weighted value of attribute in the dimension to determine the user list (UL) with similar preferences. In the second stage, the user list (UL) produced from stage 1 is used as the subject of comparison in multidimensional collaborative filtering recommendation. Dimensionality reduction (Adomavicius et al., 2005) is used to handle the ratings of users. It is also used to analyze the relationship of target users and similar user list to find the target items for the recommendation list (RL). The last stage determines the key dimensions. It uses the multi-facet to demonstrate the properties of key dimensions as the explanation of the recommendation result.

\subsection{Profile Database and Rating Database}

The profile database stores the profiles of users and contextual dimensions (time, location, companion, etc.) including properties, characteristics and operation methods. The rating database records the ratings of the users toward an item. It is considered as an important reference of the system to deduce rating.

\subsection{Movie Database}

This study establishes a movie recommender system environment that considers five contextual information namely user, movie, time, location and companion as the 
multidimensional recommendation foundation. Because the hierarchy ratings have a problem of many rating selections, therefore, this study assumes time, location and companion as a single value attribute. The movie dimension could be established to include multi-value or hierarchical attributes. The multi-facet revealed to be able to provide a hierarchical rating selection. The definitions of the five dimensions are:

(i) User: user is represented by $u$. User properties include static and dynamic properties.

(ii) Movie: movie is represented by i. It represents the recommendation target as the items indicated in the above which include multiple movie attributes.

(iii) Time: time is represented by t. It is description of the time the movie might be watched including weekdays, weekends and premiere week.

(iv) Location: location is represented by $\mathrm{p}$. The location dimension includes where the movie is watched, in a movie house or at home.

(v) Companion: companion is represented by $\mathrm{f}$. It represents the relationship between the companion and the user.

\section{Foundation of Multidimensional Recommendation}

The characteristics proposed by Adomavicius et al. (2005) as the foundation of the multidimensional recommendation system. The MD model includes the four basic characteristics and capabilities: (1) multiple dimensions, (2) profiling capabilities, (3) aggregation capabilities, and (4) multi-facet capabilities. To have a good recommendation capability in the multidimensional recommendation model, this study uses four characteristics and capabilities as the foundation of the multidimensional recommendation system. These four characteristics would be further discussed below.

\subsection{Multiple Dimensions}

The dimension represents the range of the recommendation space (RS). The traditional recommendation method considered only two dimensions namely user and item. The concept of multi-dimension is extended from the data warehouse and OLAP using multi-facet to view the data. If the rating computation would be used to handle the recommendation problem, traditional two-dimensional recommender system can be represented as $R:$ Users $\times$ Items $\rightarrow$ Ratings. It means that the rating was determined by user and item. The $r(u, i)$ values of two-dimensional " $U \times I$ " matrix was the inferring purpose of the recommendation and used the dimensions user and items as its foundation. This study adds the other contextual information and each context are represented by one dimension. The recommendation space (RS) is shown as $R S=D_{1} \times D_{2} \times \ldots \times D_{n}$. Each dimension has its own specific format, attributes and operation method. Its attributes are used as the main description of the dimension. It is shown as $D_{i} \subseteq A_{i 1} \times A_{i 2} \times \ldots \times A_{i j} . A_{i j}$ represents the properties included in the dimension $D_{i}$. In addition, the attributes could also be a set.

The dimension and attributes include the concept of hierarchy. In the dimensions items and time, the hierarchy of the item is based on the item classification structure (i.e. product classification). The representation method of the two-dimensional rating function is used as the foundation. The multidimensional recommendation model rating could be shown as $r\left(D_{1}, D_{2}, \ldots, D_{n}\right)$. The influencing key dimensions $\left(D_{1}, D_{2}, \ldots\right)$ are used to determine the value of the recommendation function. Users and items are the two basic dimensions that are indispensable in a multidimensional recommendation; therefore, the definition represented by the rating function is the level of likeness of a user towards an item in a context. For example, if a recommendation space includes a three dimensions user, item and time, its rating function is shown as $r(u, i, t)$. From r(Jason, tour-in-Tokyo-Japan, summer vacation) $=7$, it is shown that Jason likes to travel to Tokyo during summer vacation. It expresses the user's preference and explains the user behavior. In recommendation space, the key dimensions could be used as ratings just as if the purpose of the traditional recommender system lies within $U \times I$ matrix, the rating function $\left(r\left(D_{1}, D_{2}, \ldots, D_{n}\right)\right)$ of the MD model lies within a multidimensional matrix. For example, if the recommendation space includes user, item and time, the rating $r(u, i, t)$ lies in a three-dimensional space $U \times I \times T$. The three-dimensional recommendation space is shown in figure 2.

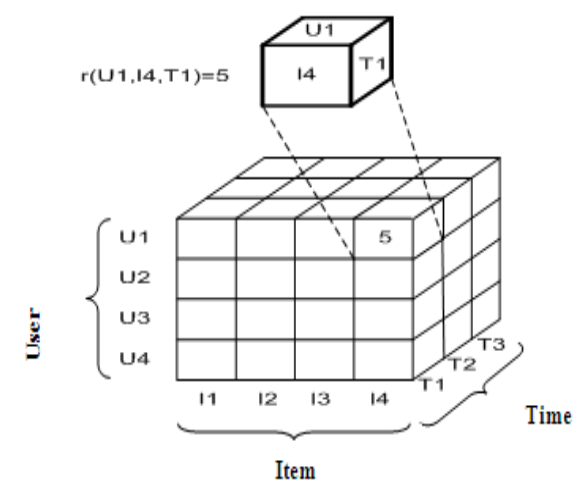

Figure2. $U \times I \times$ T Recommendation Space (Adomavicius et al., 2001)

The problem of multidimensional recommendation is similar with that of the traditional recommendation which is to calculate the rating function in the matrix. The first problem is select of dimensions. The recommendation space might include multiple dimensions but not all dimensions are applicable. In other words, not all the contextual information is influential. To select the key dimension that is most influential to the recommendation result is a really valuable topic for research. This study uses time, location and companion as the key dimensions. After analyzing the contextual information, many other interesting problems are discovered. Aside from explaining the problems from many 
different angles, analysis operations could be used to make appropriate recommendations.

\subsection{Profiling Capabilities}

This study applies the concept of profile construction in the multidimensional recommendation model. Traditional profile construction techniques are mostly applied in describing the characteristics of users and items. When applied in the multidimensional recommendation environment, there are also changes in the establishing capabilities and patterns of the profiles. The technique for establishing the multidimensional profile is more complicated and considers more problems than the traditional one. According to its capabilities of the multidimensional profiling techniques shown above, this study believes that it would surely provide better recommendation results. The multidimensional recommendation model applies more appropriate dimensional profiles as recommendation foundation. One of its advantage is it can provide appropriate and accurate recommendation results. Considering contextual information not only helps increase the accuracy of the recommendation result, it is even better for establishing rules to explain the preferences of users with special or unique demands. In addition, another advantage is that the results by the profiling can secure and reduce the recommendation range.

\subsection{Aggregation Capabilities}

Aggregation is the main purpose of establishing data cubes in data warehousing. Aggregation is a tool of the traditional analysis. When the dimensions of the data cube increase, it would affect the time of inquiry. By using aggregation, it would focus its analysis on the main information. This study combines the hierarchical structure and the aggregation capabilities for the MD model to have similar storing capability like data warehouse, OLAP data analysis and aggregation capabilities. Using movie recommendation as the example, one-on-one rating was used in the past. Using the hierarchy concept and aggregation, $\mathrm{R}$ (Tom, The Ring) $=6$ was also similar to the results $\mathrm{r}$ (Tom, sci-fi) $=6$ or $\mathrm{r}$ (male, The Ring) $=6$. The ratings of Tom towards science fiction (sci-fi) films could be used to estimate his level of likeness towards sci-fi films or use the ratings of the male population who already watched "The Ring" to estimate r(male, The Ring). The mathematical equation would be:

$$
\begin{gathered}
r(\text { Tom,sci-fi })=A G G R_{\text {M.type }=\text { sci-fir }} r(\text { Tom }, M), \\
M=\left\{\text { movie }_{l}, \ldots \text { movie }_{n}\right\}
\end{gathered}
$$

If Average (AVG) represents the aggregation function (Aggr), formula (1) would be:

$$
\begin{gathered}
r(\text { Tom,sci-fi })=A V G_{M . t y p e=s c i-f i} r(\text { Tom }, M), \\
M=\left\{\text { movie }_{1}, \text { movie }_{n}\right\}
\end{gathered}
$$

\subsection{Multi-Facet Capabilities}

Past recommender systems provided recommendation list according to the forecasted rating levels. It cannot effectively explain the recommendation result and also cannot explain how the recommendation was produced. Using museum collection database as an example, Hyvonen et al. (2003) proposed the use of Ontology as searching structure foundation and combined multi-facet classification for searching solution. Especially in handling searching results, Differences of Semantics were used to classify searching results and multi-facet demonstration was used to show searching results in order to conform the needs of users. If only a fixed attribute is used for aggregate computation in handling hierarchy ratings, contradicting results would often appear. This study believes that the result would not be the fault of aggregation but due to the selection of wrong attribute (type of movie) would lead to the wrong result of aggregation. Movies involve many properties or attributes. Each attribute could become the reason of selection by the users, therefore, the numerous attributes of the movies should be considered. The aggregation result shown in figure 3 discovers that the attributes "leading actor" and "year" are similar with the aggregation result. Both sides could provide an appropriate classification and explanation toward the recommendation result.

\section{Rating Estimation in Multidimensional Recommender Systems}

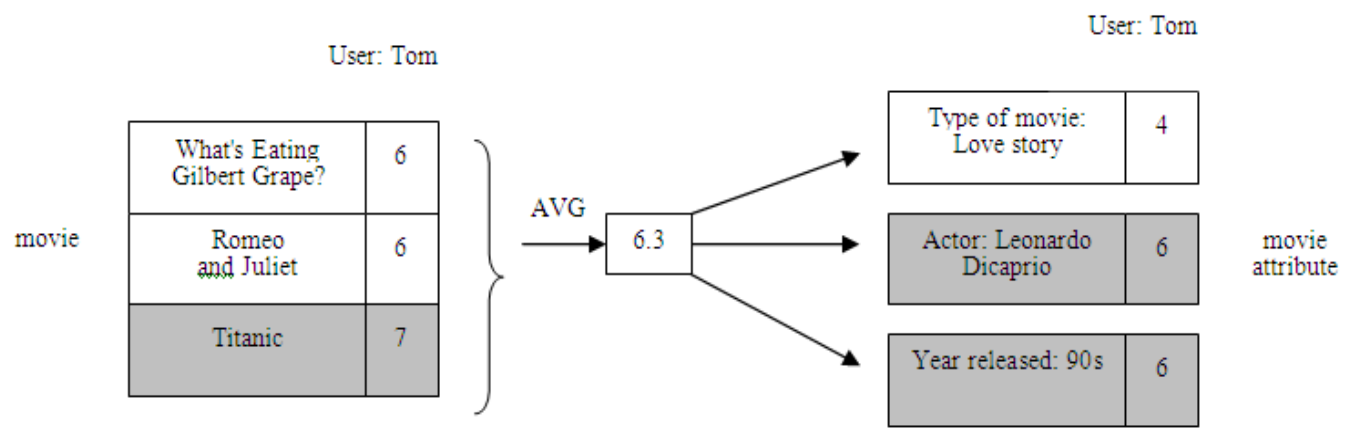

Figure3. Multi-facet Aggregation 
An important research question is how to estimate unknown ratings in a multidimensional recommendation space. As in traditional recommender systems, the key problem in multidimensional systems is the extrapolation of the rating function from a subset of ratings that are specified by the users for different levels of the aggregation hierarchies in the multidimensional cube of ratings. For example, some ratings are specified for the bottom level of individual ratings, such as John Doe assigned rating 7 to "Gladiator," - R(JD, Gladiator $)=7$, whereas others are specified for aggregate ratings, such as John Doe assigned rating 6 to action movies- $\mathrm{R}(\mathrm{JD}$, action) $=6$. Then, the general rating estimation problem can be formulated as follows:

Multi-level Multidimensional Rating Estimation Problem: given the initial (small) set of user-assigned ratings specified for different levels of the multidimensional cube of ratings, the task is to estimate all other ratings in the cube at all the levels of the OLAP hierarchies.

This rating estimation problem is formulated in its most general case, where the ratings are specified and estimated at multiple levels of OLAP hierarchies. Although there are many methods proposed for estimating ratings in traditional two-dimensional recommender systems as described, not all of these methods can be directly extended to the multidimensional case because extra dimensions and aggregation hierarchies complicate the problem. We will first describe how to estimate multidimensional ratings without aggregation hierarchies using the proposed reduction-based approach.

\subsection{An Overview of the Reduction-Based Approach}

The Reduction-Based approach reduces the problem of multidimensional recommendations to the traditional two-dimensional User $\times$ Item recommendation space. Therefore, it does not consider aggregation hierarchies and operates at the level of individual cells of the multidimensional cube of ratings described. Furthermore, one of the advantages of the reduction-based approach is that all previous research on two-dimensional recommender systems is directly applicable in the multidimensional case - any of the methods described can be applied after the reduction is done. How this reduction can be done, we consider the content presentation system discussed. Furthermore, assume that, a three-dimensional rating prediction function supporting time can be defined as -

$$
R_{\text {User } \times \text { Content } \times \text { Time }}^{D}: U \times C \times T \rightarrow \text { rating }
$$

where D contains records $<$ user, content, time, rating $>$ for the user-specified ratings. Then the three-dimensional prediction function can be expressed through a two-dimensional prediction function as follows:

$\forall(u, c, t) \in U \times C \times T, R_{U s e r \times C o n t e n t \times T i m e}^{D}(u, c, t)=R_{U \text { ser } \times \text { Content }}^{D[\text { Timet } t \mid(\text { User,Content, rating })}(u, c)$ obtained from D by selecting only those records where Time dimension has value $t$ and keeping only the corresponding values for User and Content dimensions as well as the value of the rating itself. In other words, if we treat a set of three-dimensional ratings $\mathrm{D}$ as a relation, then $\mathrm{D}[$ Time $=$ t](User, Content, rating) is simply another relation obtained from $\mathrm{D}$ by performing two relational operations: selection followed by projection.

\subsection{Combined Reduction-Based and Traditional CF Approaches}

In order to combine the two methods, we need some performance metric to determine which method "outperforms" the other one on various segments. There are several performance metrics that are traditionally used to evaluate performance of recommender systems, such as mean absolute error (MAE), mean squared error (MSE), correlation between predictions and actual ratings, precision, recall, F-measure, and the Receiver Operating Characteristic (ROC). The MAE measure is a representative example of a statistical accuracy measure. The decision-support accuracy metrics measure how well a recommender system can predict which of the unknown items will be highly rated. The F-measure is a representative example of the decision-support accuracy metric. Moreover, although both types of measures are important, it has been argued in the literature that the decision-support metrics are better suited for recommender systems because they focus on recommending high-quality items, which is the primary target of recommender systems.

After introducing the preliminary concepts, to present the combined approach that consists of the following two phases. First, using known user-specified ratings (i.e., training data), we need to determine which contextual segments outperform the traditional CF method. Second, in order to predict a rating, we need to choose the best contextual segment for that particular rating and use the two-dimensional recommendation algorithm on this contextual segment.

The combined approach is expected to perform equally well or better than the pure two-dimensional approach in practice (however, this is not an absolute theoretical guarantee, since the actual performance ultimately depends on the underlying data). The extent to which the combined approach can outperform the two-dimensional approach depends on many different factors, such as the application domain, quality of data, and the performance metric (i.e., adding contextual information to recommender systems may improve some metrics more significantly than others, as will be shown below). The main advantage of the combined reduction-based approach described is that it uses the reduction-based approach only for those contextual situations where this method outperforms the standard 2D recommendation algorithm, and continues to use the latter where there is no improvement. 


\subsection{Multi-Level Rating Estimation Problem}

Aggregate ratings, are important since they can be useful in various applications. For instance, one can estimate some of the unknown individual ratings in terms of the known aggregate and known individual ratings. Another reason for using aggregate ratings is that, under certain assumptions, they can have smaller estimation errors than individual ratings. To see this, consider the two-dimensional case of User $\times$ Item when the rating estimation function is modeled as

$$
\boldsymbol{R}_{c}(u, i)=\boldsymbol{R}_{a}(u, i)+\varepsilon\left(\mu, \sigma^{2}\right)
$$

where $\mathrm{R}_{\mathrm{a}}(\mathrm{u}, \mathrm{i})$ is the true rating of item i made by user $u, \mathrm{R}_{\mathrm{c}}(\mathrm{u}$, $i)$ is its estimated value, and $\varepsilon(\mu, \sigma 2)$ is an error term that is represented as a random variable having an unspecified distribution with mean $\mu$ and variance $\sigma 2$, and that this distribution is the same across all the users and items.

It is not true in general that estimations of aggregate ratings are always more accurate than estimations of individual ratings. Obviously, this depends on various factors, including:

(i) the rating estimation function,

(ii) the rating aggregation function (e.g., $\mathrm{AVG}$, AVG-of-Top-k, etc.), and

(iii) the accuracy measure (mean absolute error, mean squared error, F-measure, etc.) that are being used in the model.

Therefore, an important research problem would be to determine when it is the case-under what conditions an estimated aggregate rating is more accurate than the individual estimated ratings. This question also constitutes an interesting topic for future research.

\section{Experiment Design}

This study applies the proposed multidimensional recommendation in the movie recommendation. Even though most of movie information websites have detailed information of the movie, ratings, discussions, etc., it still doesn't have the capability of collecting related contextual information. Therefore, this experiment couldn't directly use the rating information of the movie information websites for analysis. Due to this problem, this study designs an experimental website and collects the necessary information to be used in this experiment. To verify the feasibility of its structure, this study makes an experimental movie recommendation system to collect the rating of the users toward the movies and provides movie recommendation according to the recorded rating. Because this study mainly focuses its discussion on the capability of the multidimensional recommendation model and not in the learning mechanism towards improving the outcome of the recommendation result, the capability of the learning mechanism is not added in the experiment process. The experimental system consists of two main capabilities namely movie rating and movie recommendation. The system structure could be divided into four parts, shown in figure 4. Detailed explanations of the parts are explained below:

Database: stores profile information of the users, movie information, actor and director information, rating information, recommendation information and statistics information.

Profiling module of users: includes four profiles namely movie, time, location and companion. The user should establish his/her personal profile at the beginning of the experiment.

Movie rating module: This study uses the collection of Truemovie.com from January 2003 to February 2006 (a total of 309 movies' information) as the experiment foundation to provide picture and text movie information for users to rate the movies. Points 1-7 are used to rate the user's interest in the movie where 5 and above representing the user is interested in that movie. It also records the user's appreciation towards the contextual information of the movie.

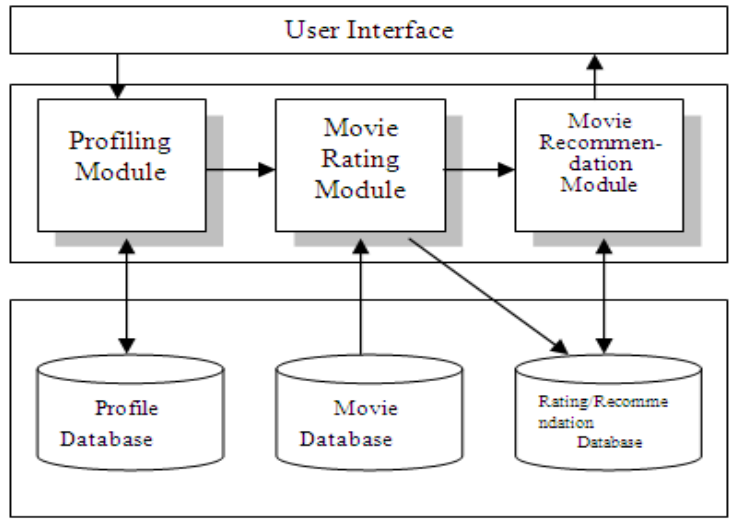

Figure4. Structure of experiment system

Movie recommendation module: The movie recommendation module is divided into two parts. The first part considers the multidimensional recommendation model of the contextual information. Firstly, it analyzes a possible movie list for recommendation according to the profile of the target customer/user. The movies in the movie list that could be recommended are declared as target movies. Users with much variation are eliminated according to the profiles of the target users and search for target users that might be in similar user list of common rating behavior. The similarity of the target user and possible similar user group are computed. If the similarity value is 0.5 greater than the threshold value, include it in similar user group. Then list the similar user list according to the rating of the target movie one by one. If similar rating of a target movie exceeds three persons, then the forecasted rating of the movie can be calculated. Provide recommendation result explanation after comparing the forecasted rating and the aggregated rating of the movie attribute. Lastly, list the top five recommended movies. The second part is the traditional collaborative filtering recommendation module. The effect of the contextual 
information is eliminated and just considers the dimensions, user and movie. List the top five recommended movies.

\subsection{Setting of System Parameters}

Before continuing with the experiment, first let's provide a clear setting of the multidimensional recommendation algorithm and the related parameters used in the experiment operation. Table1 shows the system parameter settings and their description.

Table1: Table of system parameter setting

\begin{tabular}{|c|c|}
\hline Parameter Setting & Description \\
\hline Number of experiment sample in stage 1: 40 persons & Collect movie rating information \\
\hline Number of experiment sample in stage $2: 25$ persons & $\begin{array}{l}\text { Ask } 25 \text { persons to rate the movies, render them to the multidimensional movie } \\
\text { recommendation result, provide the ratings and select its level of satisfaction }\end{array}$ \\
\hline Threshold value of the movie rating in stage $2: 25$ movies & $\begin{array}{l}\text { Set the lowest allowable value for movie rating of a user. Ensure that the common rating is } \\
\text { greater than the threshold value }\end{array}$ \\
\hline Threshold value of the liked movie: 5 & $\begin{array}{l}\text { Points } 1-7 \text { is used to rate the user's interest in the movie where } 5 \text { and above representing the } \\
\text { user is interested in that movie }\end{array}$ \\
\hline $\begin{array}{l}\text { Common rating of the movies: } \\
6 \text { movies and above }\end{array}$ & $\begin{array}{l}\text { The target user c and the possible similar user c' should have } 6 \text { or more movies with common } \\
\text { ratings to be able to compute for the similarity value }\end{array}$ \\
\hline Threshold value of the similar users: 3 and above & $\begin{array}{l}\text { To be able to compute for the forecasted rating, the number of similar target users should be } \\
\text { greater than } 3\end{array}$ \\
\hline $\begin{array}{l}\text { Similarity's threshold value: } \\
0.5 \text { and above }\end{array}$ & $\begin{array}{l}\text { To compute for the similarities between the target user } u \text { and possible similar users } u \text {, } \\
\text { greater than } 0.5 \text { could already determine the similar users }\end{array}$ \\
\hline
\end{tabular}

\subsection{Process of the Experiment}

The experiment process of this study is shown below: (i) The first stage of the experiment uses the collected user ratings as the main purpose of the recommendation computation foundation of stage two. The members of a movie discussion area are used as the experiment sample. The system in stage one doesn't recommend any movies. A total of 40 persons are examined and 1452 movie rating information is collected.

(ii) The number of examined sample in stage two is 25 and they are different from the 40 samples selected in stage one. The users are set to rate at least 25 movies before proceeding to the other parts of the experiment. The system produces two groups of recommendation results. A total of 10 movies are divided equally into 5 multidimensional recommendation results and 5 collaborative filtering recommendation results. The users separately rate their satisfaction according to the recommendation result and recommendation description The satisfaction level of the recommendation result is divided into 5 points and they could select whether they have watched the movie or not. The recommendation description is divided into 7 points.

\section{Conclusion}

This study defines the attributes and capabilities of the multidimensional recommendation structure namely multiple dimensions, profiling capabilities, aggregation capabilities and multi-facet capabilities.

Aside from proposing theories, this study designs a movie recommender prototype system to enhance the confirmation of the multidimensional recommendation result. The research results confirm that adopting contextual multidimensional recommendation method could promote the accuracy of the recommendation results. The forecasted ratings and the actual user satisfactory level are quite close meaning that the recommendation method proposed by this study is good. Besides, most of the viewing behaviors of the users are consistent with the system recommendation results which show that the recommendation result conform with the users' behaviors. In a short period of time, this study could control the preferences and interests of the users to produce good recommendation results.

Although this study and the study of Adomavicius et al. (2005) both discussed the capabilities of the multidimensional recommendation method, the purposes are different. The research purpose and emphasis of Adomavicius et al. (2005) was to propose an algorithm that descent and select the best key dimension and attributes while the purpose of this study is to propose a complete multidimensional recommendation structure and process and proposes to solve the contradicting problem of hierarchy rating using multi-facet capabilities. Although the experiment didn't focus on testing and measuring feedback and learning module, this study believes that if the module is added in the recommender system and with continuous collection of user rating information, the system could re-compute for the similarity of users and correct the user preferences then re-compute the recommendation result. Therefore, new recommendation information could be attained after a short period of time. Because the system combines multi-facet attributes, aside from producing recommendation results according to the special preferences of the users, result classification could also be done 
according to the reason of recommendation and it can even provide recommendation by immediately combining the contextual information. In addition, the following suggestions are proposed for practical application:

(i) To understand E-commerce and general consumption needs and behaviors of users in order to obtain personalization, the influence of the contextual information should be given more importance. Understand and use the followed contextual information of the consumption, browsing and rating behaviors of the users to get closer to the thoughts of the users. If necessary, without disturbing the users, user's profiles could also be used to collect related contextual information

(ii) The factors used in the actual application of the recommender system in different domains are not necessarily similar. If the concept of multi-dimension is adopted in the integration of many recommendation methods, the differences among the applications of different domains could be seen. Selecting correct method and appropriate dimensions could help obtain appropriate recommendations and support the decisions of the users.

(iii) In reality, selecting appropriate context as basis could be difficult especially if the contextual information of the domain is not shown using structure or it cannot be easily classified. Therefore, it is suggested that in analyzing the key context, enough samples should be collected to effectively measure and discover the importance of the contextual information. This characteristic is similar to the situation of digging out the key dimensions in data mining. The more information the data warehouse possesses, the more helpful it would be for the analysis and results.

\section{References}

[1] Adomavicius, G. \& Tuzhilin, A. 2001. Extending recommender systems: A multidimensional approach. Proceedings of the International Joint Conference on Artificial Intelligence (IJCAI-01), Workshop on Intelligent Techniques for Web Personalization, Seattle.

[2] Adomavicius, G., Sankaranarayanan, R., Sen, S. \& Tuzhilin, A. 2005. Incorporating Contextual Information in Recommender Systems Using a Multidimensional Approach, ACM Transactions on Information Systems, 23(1), 103-145.

[3] Baltrunas, Linas, et al. "Context relevance assessment and exploitation in mobile recommender systems." Personal and Ubiquitous Computing 16.5 (2012): 507-526.

[4] Baltrunas, Linas. "Exploiting contextual information in recommender systems."Proceedings of the 2008 ACM conference on Recommender systems. ACM, 2008.

[5] Balabanovic, M. \& Shoham, Y. 1997. Fab: Content-based, Collaborative Recommendation, Communications of the $A C M, 40(3), 66-72$.

[6] Basu, C., Hirsh, H. \& Cohen, W. 1998. Recommendation as Classification: Using Social and Content-based Information in Recommendation, Proceedings of the $15^{\text {th }}$ National Conference on Artificial Intelligence, Madison, pp. 714-720.
[7] Billsus, D. \& Pazzani, M. J. 1998. Learning Collaborative Information Filters, Proceedings of the $15^{\text {th }}$ International Conference on Machine Learning, San Francisco, pp. 46-54.

[8] Breeze, J. S., Heckerman, D. \& Kadie C. 1998. Empirical analysis of predictive algorithms for collaborative filtering, Proceedings of the $14^{\text {th }}$ Conference on Uncertainty in Artificial Intelligence, Madison, pp.43-52.

[9] Brown, P. J., Bovey, J. D. \& Chen, X. 1997. Context-aware Applications: From the Laboratory to the Marketplace, Proceedings of IEEE Personal Communications, 4(5), $58-64$.

[10] Goldberg, D., Nichols, D., Oki, B. \& Terry, D. 1992. Using collaborative filtering to weave an information Tapestry, CACM, 35(12), 61-70.

[11] Herlocker, J. L. \& Konstan, J. A. 2001. Content-Independent Task-Focused Recommendation, IEEE Internet Computing, $5(6), 40-47$

[12] Herlocker, J. L., Konstan, J., Terveen, L. \& Riedl, J. 2004. Evaluating Collaborative Filtering Recommender Systems, ACM Transactions on Information Systems, 22(1), 5-53.

[13] Karatzoglou, Alexandros, Xavier Amatriain, Linas Baltrunas, and Nuria Oliver. "Multiverse recommendation: n-dimensional tensor factorization for context-aware collaborative filtering." In Proceedings of the fourth ACM conference on Recommender systems, pp. 79-86. ACM, 2010.

[14] Liu, Liwei, et al. "Using context similarity for service recommendation."Semantic Computing (ICSC), 2010 IEEE Fourth International Conference on. IEEE, 2010.

[15] Li, Y., Lu, L. \& Xuefeng, L. 2005. A hybrid collaborative filtering method for multiple-interests and multiple-content recommendation in E-Commerce, Expert Systems with Applications, 28(1), 67-77.

[16] Linden, G., Smith, B. \& York, J. 2003. Amazon.com Recommendations Item to Item Collaborative Filtering, IEEE Internet Computing, 7(1), 2003, 76-80.

[17] Pazzani, M. 1999. A Framework for Collaborative, Content-Based and Demographic Filtering, Artificial Intelligence Review, 13(5-6), 393-408.

[18] Panniello, Umberto, et al. "Experimental comparison of pre-vs. post-filtering approaches in context-aware recommender systems." Proceedings of the third ACM conference on Recommender systems. ACM, 2009.

[19] Ricci, Francesco, Lior Rokach, and Bracha Shapira. "Introduction to recommender systems handbook." Recommender Systems Handbook. Springer US, 2011. 1-35.

[20] Said, Alan. "Identifying and utilizing contextual data in hybrid recommender systems." Proceedings of the fourth ACM conference on Recommender systems. ACM, 2010.

[21] Sarwar, B., Karypis, G., Konstan, J. \& Riedl, J. 2001. Item-based Collaborative Filtering Recommendation Algorithm, Proceedings of the $10^{\text {th }}$ International $W W W$ Conference, Hong Kong, pp. 285-295.

[22] Shih, Y. Y. \& Liu, D. R. 2005. Hybrid Recommendation Approaches: Collaborative Filtering via Valuable Content Information, Proceedings of $38^{\text {th }}$ Hawaii International 
Conference on System Sciences, Big Island.

[23] Tso-Sutter, Karen HL, Leandro Balby Marinho, and Lars Schmidt-Thieme. "Tag-aware recommender systems by fusion of collaborative filtering algorithms."Proceedings of the 2008 ACM symposium on Applied computing. ACM, 2008.

[24] Woerndl, Wolfgang, Christian Schueller, and Rolf Wojtech. "A hybrid recommender system for context-aware recommendations of mobile applications. " Data Engineering Workshop, 2007 IEEE 23rd International Conference on.
IEEE, 2007.

[25] Yang, W., Wang, Z. \& You, M. 2004. An Improved Collaborative Filtering Method for Recommendations Generation, Proceedings of the 2004 IEEE International Conference on Systems, Man and Cybernetics, The Hague, Vol. 5, pp. 4135-4139.

[26] Yujie, Zhang, and Wang Licai. "Some challenges for context-aware recommender systems." Computer Science and Education (ICCSE), 2010 5th International Conference on. IEEE, 2010. 